\title{
An Efficient Super-Resolution DOA Estimator Based on Grid Learning
}

\author{
Zhenyu $W E I^{1}$, Xin $L I^{2}{ }^{2}$ Ben $W A N G{ }^{1}$,Wei WANG ${ }^{1}, Q i L I U^{3}$ \\ ${ }^{1}$ College of Automation, Harbin Engineering University, Harbin, 150001, China \\ ${ }^{2}$ Engineering Training Center, Harbin Engineering University, Harbin, 150001, China \\ ${ }^{3}$ Dept. of Electronic Engineering, City University of Hong Kong, Hong Kong, China \\ g-weizhenyu@hrbeu.edu.cn, xinxin_forever@126.com, \\ wangben@hrbeu.edu.cn,wangwei407@hrbeu.edu.cn, qiliu47-c@my.cityu.edu.hk
}

Submitted June 25, 2019 / Accepted September 2, 2019

\begin{abstract}
Direction-of-arrival (DOA) estimation based on sparse signal reconstruction (SSR) is always vulnerable to off-grid error. To address this issue, an efficient superresolution DOA estimation algorithm is proposed in this work. Utilizing the Taylor series expansion, the sparse dictionary matrix is constructed under the off-grid model. Then, a polynomial optimization function is established based on the orthogonality principle. By minimizing the given objective function, we derive an efficient closed-form solution of the off-grid errors. Using the estimated off-grid errors, the discretized grid can be iteratively learned and approaches the true DOAs. With the newly learned grid, accurate DOA estimations can be achieved through the SSR scheme. The proposed algorithm converges fast and achieves precise DOA estimations even the step size of the discretized grid is large. The superior performance of the proposed algorithm is demonstrated by the simulation results.
\end{abstract}

\section{Keywords}

Direction of arrival (DOA) estimation, grid learning, sparse signal reconstruction (SSR), off-grid model

\section{Introduction}

Direction-of-arrival (DOA) estimation is one of the most significant array signal processing techniques which has wide range of applications, such as navigation, wireless communication, radar and sonar [1]. In the past few decades, conventional DOA estimators have been proposed including Capon [2], MUSIC [3] and ESPRIT [4]. These subspace based estimators often rely on the statistical properties of the sampled data, thus a large number of snapshots are required for precision estimation. With the development of sparse signal reconstruction (SSR) [5], [6], several SSRbased DOA estimation methods exploit the spatial sparse property of direction information to further improve the estimation performance [7-12], and these methods show strong robustness at the cases of limited snapshots.

However, SSR-based DOA estimation algorithms always require a pre-specified discretized spatial grid and suppose the true DOAs lie on the discretized grid points. In practice, this assumption cannot always be guaranteed and it will lead to discretization error, which is referred as off-grid error or basis mismatch. Furthermore, SSR-based DOA estimation algorithms will suffer from degraded performance significantly due to the off-grid error. Therefore, dense discretized grid or grid refinement operation [13] is necessary to suppress the off-grid error [14] where the computational complexity is demanding. Moreover, it still cannot guarantee that the true DOAs lie on the grid and will lead to a highly coherent dictionary matrix which violates conditions for sparse signal reconstruction according to the restricted isometry property (RIP) [15].

The effect of off-grid problem for the SSR-based algorithm has been studied in [14], which verifies that the grid mismatch will significantly affect the high-resolution spectrum analysis. Thus, various DOA estimators have been proposed to account for the off-grid error [16-18]. In [16], the sparse total least squares (S-TLS) algorithm is proposed which has been approved to be optimal in the maximum a posteriori (MAP) sense. However, the S-TLS algorithm achieves optimal solution under the assumption that the perturbation caused by the dictionary mismatch follows Gaussian distribution. It has been shown in [17] that the Gaussian distribution cannot be fully satisfied within the off-grid problem. To deal with this issue, an off-grid model based on the Taylor series expansion is established and the corresponding off-grid sparse Bayesian inference (OGSBI) algorithm is designed under the assumption of off-grid error following uniformly distribution in a bounded interval [17]. The OGSBI algorithm achieves superior performance and converges faster than the S-TLS algorithm. A non-convex optimization based DOA estimation algorithm is presented in [18] which estimates 
DOAs with a two-step iterative method. However, as these algorithms aforementioned [17], [18] always rely on the accuracy of the steering vector approximation, their estimation performance degrade in the case of coarse grids. Hence a root sparse Bayesian learning (RSBL) method is developed in [19] by refining the grids from the root of certain polynomial, and a super-resolution iterative reweighted algorithm is proposed using convex relaxation with the log-sum sparsity-encouraging function [20]. Nevertheless, the iterative reweighted algorithm is limited by the heavy computational complexity due to the alternating optimization.

On the other hand, the atomic norm and covariance matching criteria have been applied into gridless DOA estimation [21], [22]. Utilizing the atomic norm and covariance matching criteria, the off-grid DOA estimation problem can be transferred into a semi-definite programming (SDP) problem via reconstructing Toeplitz matrices of direction information. Unluckily, the SDP problem suffers from heavy computational load [23].

In this paper, an efficient super-resolution DOA estimator based on the grid learning scheme is proposed. We first construct the sparse dictionary matrix based on the off-grid model with the Taylor series expansion. As for the orthogonal characteristic between the steering vectors and the noise subspace, a polynomial optimization objective function is proposed, and then the resulting closed-form solution with respect to the off-grid error is derived. With the estimation of the off-grid error, the discretized grids are continually learned and revised along the true DOAs. After several iterations, a non-uniform discretized grid dictionary is established. It should be mentioned that the number of discretized grid does not change throughout the iterative process. Thus, by using the newly learned grids, the accuracy DOA estimations can be obtained efficiently. The proposed algorithm converges fast and achieves Cramér-Rao lower bound (CRLB) [24] asymptotic performance even the step size is large. Simulation results verify the effectiveness and accuracy of the proposed algorithm.

The main advantages of the proposed algorithm are categorized as follows: 1) we derive an available closed-form solution for the off-grid DOA estimation problem with the advantage of low computational complexity. 2) The proposed algorithm can converge fast even when the grid step size of the sparse dictionary is large. 3) The grid is fixed and learned iteratively with the estimation of the grid errors.

The outline of this paper is organized as follows. In Sec. 2, the signal model is built. Section 3 contains two parts, we review the off grid problem exploiting the Taylor series expansion in the first part, then in the second part, a super-resolution DOA estimation algorithm is elaborated. Section 4 presents the results of numerical simulations. The conclusion is provided in Sec. 5.

Notations: Lower-case and upper-case bold characters are used to denote vectors and matrices, respectively. $\mathbf{I}_{N}$ denotes the $N \times N$ identity matrix. $(\cdot)^{\mathrm{T}}$ and $(\cdot)^{\mathrm{H}}$ denote the transpose and Hermitian operator, respectively. E[·] stands for an expectation operator. $\operatorname{diag}\{\mathbf{x}\}$ denotes a diagonal matrix with the elements of $\mathbf{x}$ constituting the diagonal entries. $\mathfrak{R}\{\cdot\}$ stands for the real part of complex variable. In addition, $\|\cdot\|_{2}$ denote the $\ell_{2}$-norm. $\mathbb{C}^{M \times N}\left(\mathbb{C}^{M}\right)$ stands for an $M \times N$ $(M \times 1)$ complex matrix (vector) set.

\section{Signal Model}

Consider $K$ uncorrelated far-field narrow-band signals from directions $\boldsymbol{\theta}=\left[\theta_{1}, \ldots, \theta_{K}\right]^{\mathrm{T}}$ impinging on a linear array consisted of $M$ antennas, where $\theta_{k}$ is random sampled from the continuous angle domain $\left(-\frac{\pi}{2}, \frac{\pi}{2}\right)$. The complex-valued baseband received signal at time index $t$ can be expressed as

$$
\mathbf{x}(t)=\sum_{k=1}^{K} \mathbf{a}\left(\theta_{k}\right) s_{k}(t)+\mathbf{n}(t)=\mathbf{A}(\boldsymbol{\theta}) \mathbf{s}(t)+\mathbf{n}(t)
$$

where $\mathbf{A}(\boldsymbol{\theta})=\left[\mathbf{a}\left(\theta_{1}\right), \cdots, \mathbf{a}\left(\theta_{K}\right)\right] \in \mathbb{C}^{M \times K}$ is the array manifold matrix and $\mathbf{a}\left(\theta_{k}\right)$ is the steering vector corresponding to the $k$ th signal. $\mathbf{s}(t)=\left[s_{1}(t), \cdots, s_{K}(t)\right]^{\mathrm{T}} \in \mathbb{C}^{K}$ is the signal waveform vector and $\mathbf{n}(t)=\left[n_{1}(t), \cdots, n_{M}(t)\right]^{\mathrm{T}} \in \mathbb{C}^{M}$ is the white additive complex Gaussian noise term with zero-mean and variance matrix $\sigma_{n}^{2} \mathbf{I}_{M}$. In addition, we assume that $\mathbf{s}(t)$ is statistically independent of the noise term $\mathbf{n}(t)$. With $T$ collected samples, the received signal matrix is written as

$$
\mathbf{X}=[\mathbf{x}(1), \cdots, \mathbf{x}(T)]=\mathbf{A}(\boldsymbol{\theta}) \mathbf{S}+\mathbf{N}
$$

where $\mathbf{S}=[\mathbf{s}(1), \cdots, \mathbf{S}(T)]$ and $\mathbf{N}=[\mathbf{n}(1), \cdots, \mathbf{n}(T)]$. The sampling covariance matrix of $\mathbf{X}$ is calculated as $\hat{\mathbf{R}}=$ $(1 / T) \mathbf{X X} \mathbf{X}^{\mathrm{H}}$. The eigendecomposition of $\hat{\mathbf{R}}$ can be expressed as

$$
\hat{\mathbf{R}}=\hat{\mathbf{E}}_{s} \hat{\boldsymbol{\Sigma}}_{s} \hat{\mathbf{E}}_{s}^{\mathrm{H}}+\hat{\mathbf{E}}_{n} \hat{\boldsymbol{\Sigma}}_{n} \hat{\mathbf{E}}_{n}^{\mathrm{H}}
$$

where $\hat{\mathbf{E}}_{s}$ is the signal subspace corresponding to the $K$ largest eigenvalues contained in $\hat{\boldsymbol{\Sigma}}_{s}=\operatorname{diag}\left\{\left[\sigma_{1}^{2}, \cdots, \sigma_{K}^{2}\right]\right\}$. In addition, $\hat{\mathbf{E}}_{s}$ expands the same subspace with the array manifold matrix $\mathbf{A}(\boldsymbol{\theta})$. Similarly, $\hat{\mathbf{E}}_{n}$ is the noise subspace corresponding to the $M-K$ smallest eigenvalues contained in $\hat{\boldsymbol{\Sigma}}_{n}=\operatorname{diag}\left\{\left[\sigma_{K+1}^{2}, \cdots, \sigma_{M}^{2}\right]\right\}$.

\section{Super-Resolution DOA Estimation Algorithm}

In this section, the off-grid problem is first reviewed. Then, the proposed super-resolution DOA estimation algorithm is developed.

\subsection{Off-Grid Problem}

Denote $\boldsymbol{\Theta}=\left[\phi_{1}, \cdots, \phi_{L}\right]$ as an $L$-element uniformly discretized spatial grid with step size $\tau$ in the range of $[-\pi / 2, \pi / 2]$. Thus, an overcomplete dictionary $\overline{\mathbf{A}}(\boldsymbol{\Theta})=\left[\mathbf{a}\left(\phi_{1}\right), \cdots, \mathbf{a}\left(\phi_{L}\right)\right]$ is constructed readily. In the offgrid scenario, the true DOAs do not lie on the grid points, 
i.e., $\theta_{k} \notin \boldsymbol{\Theta}$ with $k=1, \cdots, K$. Let $\beta_{k}$ denote the off-grid error defined as $\beta_{k}=\theta_{k}-\phi_{l_{k}}, l_{k} \in\{1, \ldots, L\}$, and $\phi_{l_{k}}$ is the nearest grid point to the true DOA $\theta_{k}$. Utilizing the firstorder Taylor series expansion, the approximation of the true steering vector $\mathbf{a}\left(\theta_{k}\right)$ can be expressed as

$$
\mathbf{a}\left(\theta_{k}\right) \approx \mathbf{a}\left(\phi_{l_{k}}\right)+\mathbf{b}\left(\phi_{l_{k}}\right) \beta_{k}
$$

where $\mathbf{b}\left(\phi_{l_{k}}\right)=\partial \mathbf{a}\left(\phi_{l_{k}}\right) / \partial \phi_{l_{k}}$. Motivated by this fact, a new overcomplete dictionary matrix can be further constructed by applying the first-order Taylor series expansion

$$
\boldsymbol{\Phi}=\overline{\mathbf{A}}(\boldsymbol{\Theta})+\overline{\mathbf{B}}(\boldsymbol{\Theta}) \operatorname{diag}\{\overline{\boldsymbol{\beta}}\}
$$

where $\overline{\mathbf{B}}(\boldsymbol{\Theta})=\left[\mathbf{b}\left(\phi_{1}\right), \cdots, \mathbf{b}\left(\phi_{L}\right)\right] . \overline{\boldsymbol{\beta}}$ is a sparse vector of the off-grid error. It should be noted that the expected locations of the nonzero elements of $\overline{\boldsymbol{\beta}}$ also correspond to the DOAs.

The singular value decomposition (SVD) of $\mathbf{X}$ is expressed as $\mathbf{X}=\mathbf{U} \mathbf{\Lambda} \mathbf{V}^{\mathrm{H}}$, where $\mathbf{U}$ and $\mathbf{V}$ are left and right singular vectors, respectively. Denote $\mathbf{V}_{s} \in \mathbb{C}^{T \times K}$ as right singular vectors corresponding to the $K$ largest singular values. Multiplying the received signal matrix with $\mathbf{V}_{s}$, we have

$$
\mathbf{X}_{v}=\mathbf{X} \mathbf{V}_{s}=\mathbf{A}(\boldsymbol{\theta}) \mathbf{S}_{v}+\mathbf{N}_{v}
$$

where $\mathbf{S}_{v}=\mathbf{S} \mathbf{V}_{s}$ and $\mathbf{N}_{v}=\mathbf{N V}_{s}$. As explained in [13], $\mathbf{X}_{v}$ expands the signal subspace and both the computational complexity and the sensitivity to noise in $\mathbf{X}_{v}$ are reduced. By ignoring the approximation error, the sparse form of $\mathbf{X}_{v}$ is expressed as

$$
\mathbf{X}_{v}=\boldsymbol{\Phi} \overline{\mathbf{S}}+\mathbf{N}_{v}
$$

where $\overline{\mathbf{S}} \in \mathbb{C}^{L \times K}$ is a row-sparse matrix with $K$ nonzero rows and the expected locations of the nonzero rows correspond to the true DOAs.

\subsection{Proposed Algorithm}

As it is known, the Taylor series expansion based signal model in (4) only achieves accurate approximation when the grid points are close to the true DOAs. Thus, with the increase of the off-grid error $\beta_{k}$, especially under the large step size case, the approximation of the steering vector in (4) will be contaminated heavily which will further lead to degradation of the DOA estimation performance. This is the reason why the Taylor series expansion based off-grid DOA estimation algorithms, such as the OGSBI algorithm [17], will have degraded performance under the case of large step size. On the contrary, dense discretized grid will lead to a highly coherent dictionary matrix and heavy computational complexity.

To overcome this challenging issue, the main idea of the proposed grid learning scheme is to learn the discretized grid utilizing the off-grid error estimates. Thus, a more accurate steering vector approximation can be achieved and the revised grid points will close to the true DOA values. As a result, the performance of the sparse vector estimation will be improved significantly. Since the total number of the grid points remains constant, the computational complexity of the grid learning scheme increases slightly. The diagram of the proposed grid learning scheme is introduced in Fig. 1. From Fig. 1, it should be noted that the grid point $\phi_{l_{k}}$ is approaching the true DOA iteratively by off-grid error compensation.

Note that $\mathbf{X}_{v}$ expands the signal subspace, the true steering vector $\mathbf{a}\left(\theta_{k}\right)$ is highly correlated with $\mathbf{X}_{v}$. Mathematically, there exist $K$ atoms in $\overline{\mathbf{A}}(\boldsymbol{\Theta})$ having the largest correlation factors with $\mathbf{X}_{v}$ and the corresponding grid points are the $K$ grid points nearest to the true DOAs. The indexes set of these grid points is given as

$$
\Omega=\left\{l_{k}:\left\|\mathbf{a}^{H}\left(\phi_{l_{k}}\right) \mathbf{X}_{v}\right\|_{2}^{2}>\zeta\right\}
$$

with $l_{k} \in\{1, \cdots, L\}$ and $k=1, \cdots, K$. Since $\hat{\mathbf{E}}_{s}$ also is the signal subspace, it can be used to replace $\mathbf{X}_{v}$ in (8). In addition, $\zeta$ is a threshold parameter used to select the $K$ sparse atoms.

With the indexes set $\Omega$, the submatrice of $\overline{\mathbf{A}}(\boldsymbol{\Theta})$ and $\overline{\mathbf{B}}(\boldsymbol{\Theta})$ can be respectively constructed as

$$
\begin{aligned}
\overline{\mathbf{A}}_{\Omega} & =\left[\mathbf{a}\left(\phi_{l_{1}}\right), \cdots, \mathbf{a}\left(\phi_{l_{K}}\right)\right] \\
\overline{\mathbf{B}}_{\Omega} & =\left[\mathbf{b}\left(\phi_{l_{1}}\right), \cdots, \mathbf{b}\left(\phi_{l_{K}}\right)\right]
\end{aligned}
$$

where $l_{k} \in \Omega$. Similarly, we also denote $\overline{\boldsymbol{\beta}}_{\Omega}=\left[\beta_{l_{1}}, \cdots, \beta_{l_{k}}\right]^{\mathrm{T}}$ as a subvector of $\overline{\boldsymbol{\beta}}$. Theoretically, $\overline{\boldsymbol{\beta}}_{\Omega}$ contains $K$ nonzero elements of $\overline{\boldsymbol{\beta}}$. In this case, only $K$ parameters are required to be estimated which will reduce the computational complexity of the proposed algorithm effectively.

By utilizing the property that the signal subspace and the noise subspace are orthogonal to each other, we construct the objective function $f\left(\beta_{l_{k}}\right)$ with the steering vector approximation in (4)

$$
f\left(\beta_{l_{k}}\right)=\left[\mathbf{a}\left(\phi_{l_{k}}\right)+\mathbf{b}\left(\phi_{l_{k}}\right) \beta_{l_{k}}\right]^{\mathrm{H}} \hat{\mathbf{E}}_{n} \hat{\mathbf{E}}_{n}^{\mathrm{H}}\left[\mathbf{a}\left(\phi_{l_{k}}\right)+\mathbf{b}\left(\phi_{l_{k}}\right) \beta_{l_{k}}\right] .
$$

Thus, the off-grid error $\beta_{l_{k}}$ can be estimated by minimizing the objective function $f\left(\beta_{l_{k}}\right)$

$$
\hat{\beta}_{l_{k}}=\arg \min _{\beta_{l_{k}}} f\left(\beta_{l_{k}}\right)
$$

with $k=1, \cdots, K$.

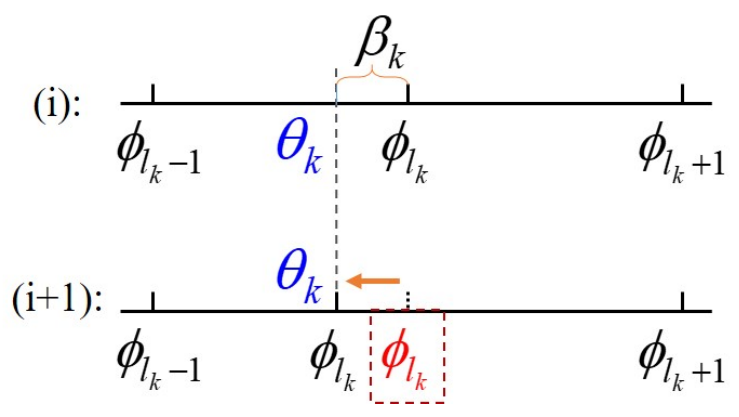

Fig. 1. Diagram of the proposed grid learning scheme. 
After formula simplification, the objective function $f\left(\beta_{l_{k}}\right)$ is rewritten as

$$
f\left(\beta_{l_{k}}\right)=c_{2, k} \beta_{l_{k}}^{2}+c_{1, k} \beta_{l_{k}}+c_{0, k}
$$

where the polynomial coefficients $c_{2, k}, c_{1, k}$ and $c_{0, k}$ are respectively expressed as

$$
\begin{aligned}
& c_{2, k}=\mathbf{b}^{\mathrm{H}}\left(\phi_{l_{k}}\right) \hat{\mathbf{E}}_{n} \hat{\mathbf{E}}_{n}^{\mathrm{H}} \mathbf{b}\left(\phi_{l_{k}}\right)=\left\|\hat{\mathbf{E}}_{n}^{\mathrm{H}} \mathbf{b}\left(\phi_{l_{k}}\right)\right\|_{2}^{2}, \\
& c_{1, k}=2 \mathfrak{R}\left\{\mathbf{a}^{\mathrm{H}}\left(\phi_{l_{k}}\right) \hat{\mathbf{E}}_{n} \hat{\mathbf{E}}_{n}^{\mathrm{H}} \mathbf{b}\left(\phi_{l_{k}}\right)\right\}, \\
& c_{0, k}=\mathbf{a}^{\mathrm{H}}\left(\phi_{l_{k}}\right) \hat{\mathbf{E}}_{n} \hat{\mathbf{E}}_{n}^{\mathrm{H}} \mathbf{a}\left(\phi_{l_{k}}\right)=\left\|\hat{\mathbf{E}}_{n}^{\mathrm{H}} \mathbf{a}\left(\phi_{l_{k}}\right)\right\|_{2}^{2} .
\end{aligned}
$$

From (12), it is clear that $f\left(\beta_{l_{k}}\right)$ is a quadratic polynomial function of $\beta_{l_{k}}$. Note that $c_{2, k}>0, f\left(\beta_{l_{k}}\right)$ achieves its minimum value when $\beta_{l_{k}}=-c_{1, k} / 2 c_{2, k}$. Combining the constraint of range of $\beta_{l_{k}}$, i.e., $\left(\phi_{l_{k}-1}-\phi_{l_{k}}\right) / 2 \leqslant \beta_{l_{k}} \leqslant$ $\left(\phi_{l_{k}+1}-\phi_{l_{k}}\right) / 2$. Thus we can derive a closed-form solution of the objective function (12) easily and the estimation of $\beta_{l_{k}}$ can be given as below

$$
\hat{\beta}_{l_{k}}=\left\{\begin{array}{lr}
-\frac{c_{1, k}}{2 c_{2, k}}, & \frac{\phi_{l_{k}-1}-\phi_{l_{k}}}{2} \leqslant \beta_{k} \leqslant \frac{\phi_{l_{k}+1}-\phi_{l_{k}}}{2}, \\
\frac{\phi_{l_{k}-1}-\phi_{l_{k}}}{2}, & -\frac{c_{1, k}}{2 c_{2, k}}<\frac{\phi_{l_{k}-1}-\phi_{l_{k}}}{2}, \\
\frac{\phi_{l_{k}+1}-\phi_{l_{k}}}{2}, & -\frac{c_{1, k}}{2 c_{2, k}}>\frac{\phi_{l_{k}+1}-\phi_{l_{k}}}{2} .
\end{array}\right.
$$

It is to be noted here that we do not need to calculate all these three coefficients in (13), where $c_{2, k}$ and $c_{1, k}$ are only needed to estimate the off-grid error $\beta_{l_{k}}$ by exploiting the closed-form solution (14).

Once $K$ nonzero elements of $\overline{\boldsymbol{\beta}}$ are obtained by solving (11), the discretized grid $\boldsymbol{\Theta}$ is revised as

$$
\boldsymbol{\Theta}^{\prime}=\boldsymbol{\Theta}+\overline{\boldsymbol{\beta}} \text {. }
$$

Since the optimal solution of (11) can be found in the given interval, it guarantees that the proposed algorithm converges within several iterations. With the newly learned grid $\boldsymbol{\Theta}^{\prime}$, accurate DOA estimation can be achieved as follows

$$
\min _{\overline{\mathbf{s}}^{\ell_{2}}}\left\|\overline{\mathbf{S}}^{\ell_{2}}\right\|_{1} \quad \text { s.t. }\left\|\mathbf{X}_{v}-\overline{\mathbf{A}}\left(\boldsymbol{\Theta}^{\prime}\right) \overline{\mathbf{S}}\right\|_{F}^{2}<\delta
$$

where $\overline{\mathbf{s}}_{l}^{\ell_{2}}=\sqrt{\sum_{k=1}^{K} \bar{S}_{l, k}^{2}}$ and $\bar{S}_{l, k}$ is the $(l, k)$ th element of $\overline{\mathbf{S}}$. $\delta$ is a user-specific tolerance factor. The SSR problem in (16) can be effectively solved using various methods, such as OMP [25], Lasso [26] and $\ell_{1}$-SVD [13].

For clarity, The main steps of the proposed algorithm is summarized as follow,

- Step 1, initialization: The initial discretized grid $\boldsymbol{\Theta}^{(0)}$, $\overline{\boldsymbol{\beta}}^{(0)}=\mathbf{0}_{L}$, the number of targets $K, r=0$ and the maximum number of iteration $\Upsilon$.

- Step 2, calculate $\hat{\mathbf{E}}_{n}$ and $\mathbf{X}_{v}$ using (3) and (6), respectively.
- Step 3, obtain the indexes set $\Omega^{(i)}$ from (8) and calculate $\hat{\beta}_{l_{k}}^{(i)}$ through (14).

- Step 4, revise the discretized grid as $\boldsymbol{\Theta}^{(i+1)}=\boldsymbol{\Theta}^{(i)}+\overline{\boldsymbol{\beta}}^{(i)}$.

- Step 5, repeat Steps 3 to 4 until maximum number of iteration is achieved or $\left|\hat{\beta}_{l_{k}}^{(i+1)}-\hat{\beta}_{l_{k}}^{(i)}\right|<10^{-8}$.

- Step 6, estimate the DOAs by solving (16).

\section{Simulation Results}

In this section, a 10-elements $(M=10)$ uniform linear array (ULA) with half-wavelength interelement spacing is adopted. In addition, we assume three uncorrelated signal sources $(K=3)$ impinging on the ULA from directions $-13.8597^{\circ}, 4.3692^{\circ}$ and $28.2096^{\circ}$, respectively. An initial discretized grid from $-90^{\circ}$ to $90^{\circ}$ is applied. All simulations in this section are performed by Matlab 2014a on a PC platform with MS Windows 10 operation system, 16 GB memory and Intel Quad-Core i7 processor. In addition, the signal to noise ratio $(\mathrm{SNR})$ is defined as $\mathrm{SNR}=10 \log _{10} \frac{E\left[\|\mathbf{s}(t)\|^{2}\right]}{\sigma_{n}^{2}}$.

In the first simulation, we provide the iteration process of grid learning. In Fig. 2, the initial discretized grid points are uniformly distributed with the grid step size of $9^{\circ}$, and the blue squares and the red dot lines represent the grid points and the positions of true targets, respectively. It is obvious that the grid points around the true DOAs are fixed and learned by iterations, and approach the more suitable grid points after about 4 iterations. It should be noted that the iterative process will not increase the number of grid points in the sparse dictionary, thus it does not increase the computational complexity of the SSR-based DOA estimation algorithm. Then a normalized spatial spectra is achieved using the OMP algorithm and the proposed super-resolution DOA estimation algorithm. The step size of the initial discretized grid is set as $\tau=9^{\circ}$. The number of snapshots is set as $T=500$ and $\mathrm{SNR}=5 \mathrm{~dB}$. From Fig. 3 , we can see that the OMP algorithm achieves poor DOA estimation due to the existence of the off-grid error. Instead, the proposed algorithm overcomes the off-grid problem and accurate DOA estimations are achieved due to the newly sparse dictionary is exploited.

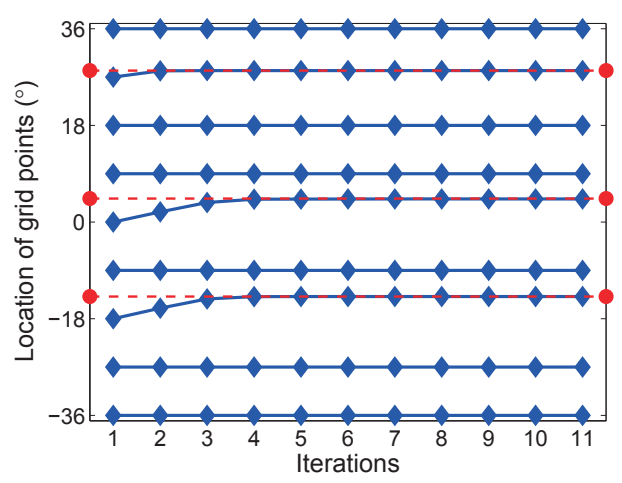

Fig. 2. Grid learning course in the proposed algorithm. 


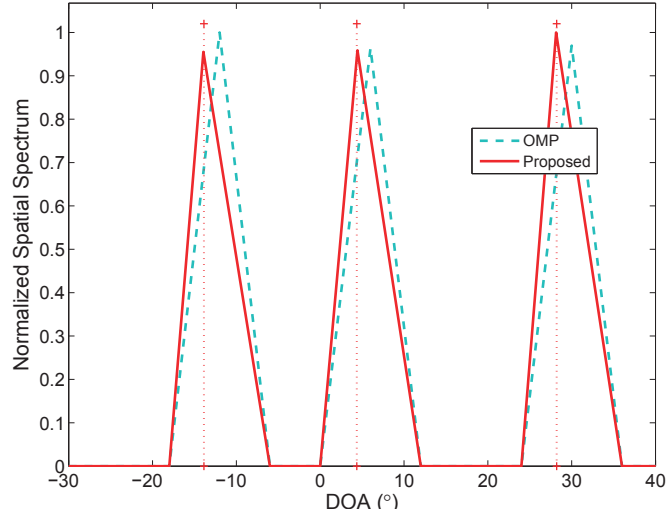

Fig. 3. The normalized spatial spectrum with the new learned grid point.

To further examine the performance of the proposed super-resolution DOA estimation algorithm, the root mean square error (RMSE) is defined as

$$
\mathrm{RMSE}=\sqrt{\frac{1}{K P} \sum_{p=1}^{P} \sum_{k=1}^{K}\left(\hat{\theta}_{k, p}-\theta_{k}\right)^{2}}
$$

where $P$ is the number of Monte Carlo trials used to calculate the RMSE. $\hat{\theta}_{k, p}$ is the DOA estimation of $\hat{\theta}_{k}$ in the $p$ th trial.

Figure 4 illustrates the RMSE comparison versus SNR of the OMP algorithm, the OGSBI algorithm [17], the RSBL algorithm [19], the SURE_IR algorithm [20], the proposed algorithm and the CRLB. In this simulation, the step size of the initial discretized grid is set as $4^{\circ}$ and the number of snapshots is $T=200$. The RMSEs are calculated among 1000 Monte Carlo trials. From these results, we can see that the proposed algorithm achieves the best DOA estimation performance among all of the algorithms. In addition, the proposed algorithm achieves CRLB asymptotic performance when SNR $>-8 \mathrm{~dB}$. Due to the off-grid error, the OMP algorithm achieves heavily degraded performance. Although the SURE_IR and RSBL algorithm show better estimation accuracy than OMP, the performance of these two algorithm are still limited due to the large grid step size. The performance of the OGSBI algorithm also degrades heavily when SNR $>10 \mathrm{~dB}$. The reason is that the large step size involves large steering vector approximation.

In order to examine the performance of the proposed DOA estimator with different initial search steps, the RMSE comparison of OGSBI, RSBL and the proposed estimator is illustrated in Fig. 5 after 1000 Monte Carlo trails, where the step sizes of initial discretized grid are set as 2, 4, 6 and 8 respectively with different markers. As shown in Fig. 5, the proposed DOA estimator shows robust performance throughout all range of the SNR with the increase of the step size. On the contrary, OGSBI and RSBL fail to provide a satisfying performance when the value of step size is large. Especially, the OGSBI algorithm has a significant degraded performance when $\tau=8$, due to the larger steering vector approximation. The results demonstrate the robustness of the proposed DOA estimator under different initial search step size $\tau$.

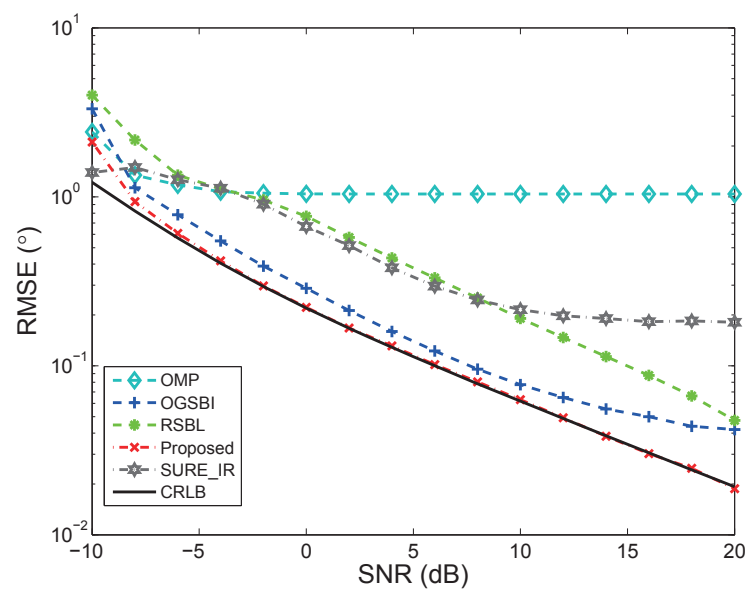

Fig. 4. RMSE versus SNR.

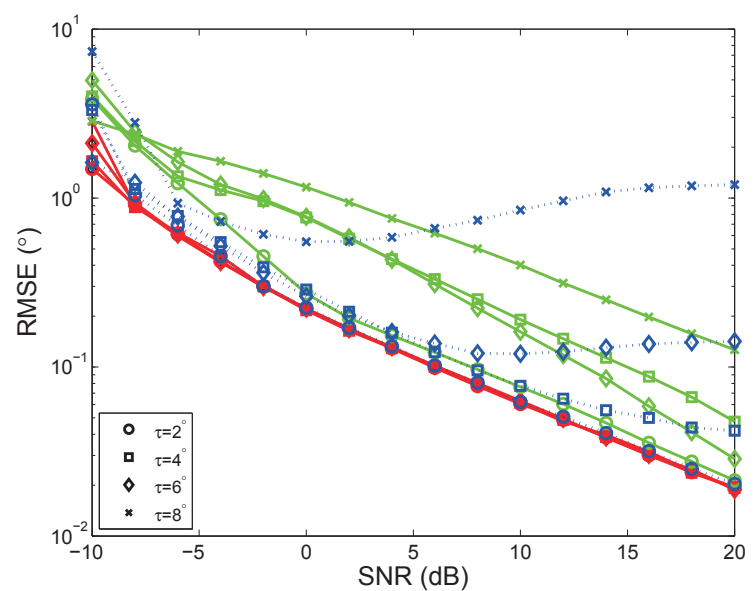

Fig. 5. RMSE versus SNR with different step sizes of the initial discretized grid. The red lines, blue dash lines and green lines denote the results achieved from the proposed algorithm, OGSBI algorithm and RSBL algorithm, respectively.

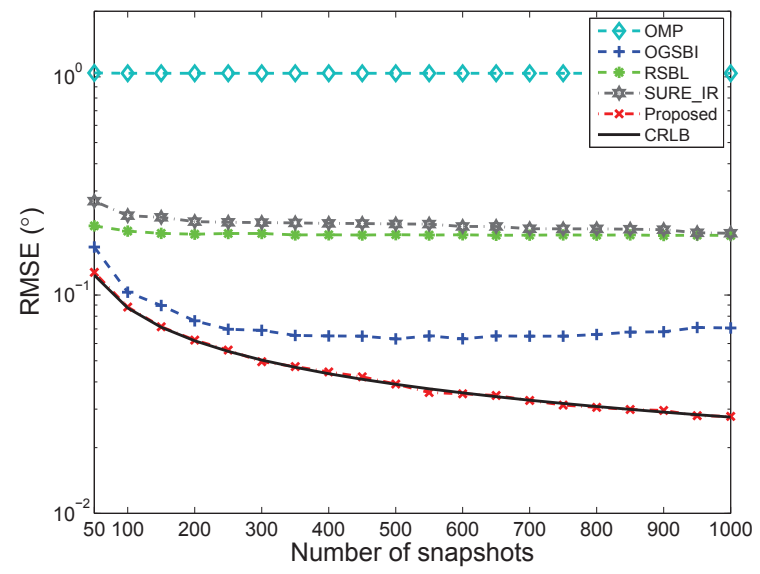

Fig. 6. RMSE versus the number of snapshots.

In the next simulation, RMSE versus the number of snapshots is examined in Fig. 6 after 1000 Monte Carlo trails. The SNR is set as $10 \mathrm{~dB}$ and the step size is set as $\tau=4^{\circ}$. Similar with the results in Fig. 4 , the proposed algorithm still achieves the best estimation performance which approaches the CRLB. Due to the existence of the off-grid error, the OMP algorithm achieves a floor performance in 
this simulation. The RSBL and SURE_IR algorithms are not sensitive to the number of snapshots. However, the OGSBI algorithms obtain a floor performance when $T \geq 300$. The reason is that the accuracy of the steering vector approximation is the main factor which limits the estimation performance in this scenario.

The convergence performance of the proposed algorithm is demonstrated in Fig. 7. The number of snapshots is $T=200$ and SNR $=10 \mathrm{~dB}$. From this result, it is clear that the proposed algorithm converges within three iterations even the step size is $\tau=8^{\circ}$. The fast convergence makes the proposed algorithm achieve robust and outstanding performance even with large value of step size.

To further verify the computational complexity of proposed DOA estimator, the CPU running time comparison of the OMP, OGSBI, RSBL, SURE_IR and the proposed estimator is plotted in Fig. 8 after 100 Monte Carlo trails. In this simulation, the number of snapshots are set as 200 as well as the step size is $\tau=4^{\circ}$. From Fig. 8, it is obviously that the proposed estimator is much more efficient than the other algorithms, except for OMP. In this simulation, we can conclude that the grid learning process is efficient and does not increase too much computational complexity compare with OMP.

In order to intuitively analyze the advantages of the proposed DOA estimator, a comparison table is provided. In Tab. 1, the RMSEs are calculated with 1000 Monte Carlo trails under the configuration of SNR $=10 \mathrm{~dB}$ and $T=200$. As shown in Tab. 1, the proposed estimator is more efficient than OGSBI, RSBL and SURE_IR algorithms, especially
SURE_IR algorithm suffers from a heavy computational load. In addition, the proposed estimator shows a robust and outstanding performance even when the step size $\tau$ is large.

\section{Conclusion}

In this paper, an efficient super-resolution DOA estimator based on the grid learning is proposed. By combining the Taylor series expansion and the orthogonal property between the signal and noise subspace, a polynomial optimization function is established and the effective closed-form solution is derived at the same time. The off-grid error is then efficiently estimated by the closed-form solution. Thus, the discretized grid is iteratively revised to make sure the grid points approach the true DOAs. At last, the DOAs are estimated accurately through the SSR scheme. The proposed algorithm converges fast and achieves CRLB asymptotic performance under the off-grid case even the grid step size is large. Simulation results demonstrate the effectiveness of the proposed super-resolution DOA estimator.

\section{Acknowledgments}

The research is funded in part by the National Natural Science Foundation $(61571148,61871143)$, Fundamental Research for the Central University (HEUCFG201823, 3072019CF0402), Heilongjiang Natural Science Foundation (LH2019F006) and Research and Development Project of Application Technology in Harbin (2017R-AQXJ095).

\begin{tabular}{|l|c|c|c|c|}
\hline Algorithms & RMSE $(\tau=4)(\circ)$ & RMSE $(\tau=6)(\circ)$ & RMSE $(\tau=8)(\circ)$ & CPU time $(s)$ \\
\hline OGSBI & 0.0773 & 0.1198 & 0.8483 & 0.0185 \\
\hline RSBL & 0.1617 & 0.1908 & 0.4016 & 0.3370 \\
\hline SURE_IR & 0.2150 & 0.2251 & 0.2083 & 1.6690 \\
\hline Proposed & 0.0605 & 0.0614 & 0.0631 & 0.0108 \\
\hline
\end{tabular}

Tab. 1. Performance comparison of different algorithms.

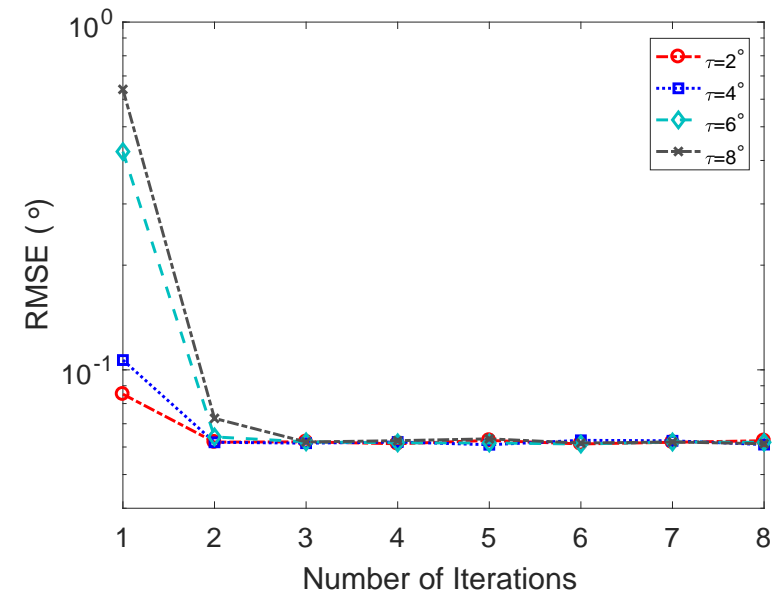

Fig. 7. Convergence performance of the proposed algorithm.

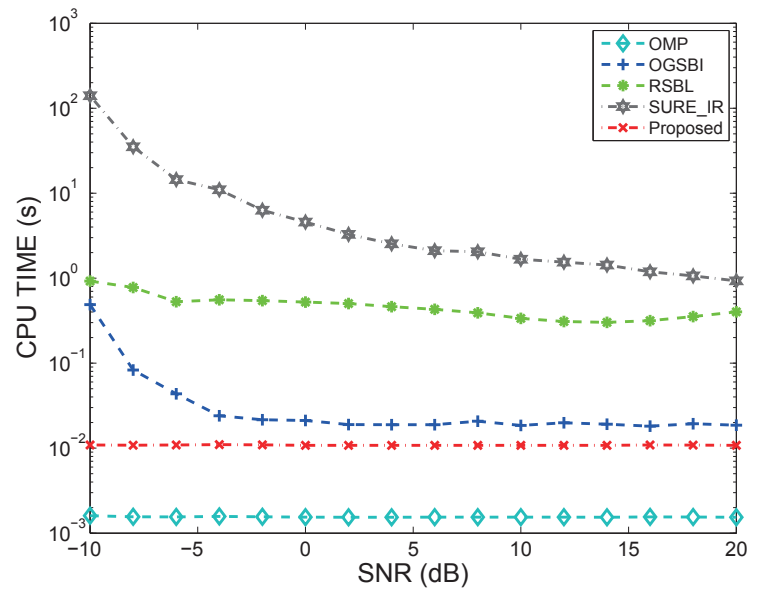

Fig. 8. CPU running time versus SNR. 


\section{References}

[1] VAN TREES, H. L. Detection, Estimation and Modulation Theory: Optimal Array Processing. 1st ed., Hoboken (USA): John Wiley \& Sons, 2004. ISBN: 0471093904

[2] CAPON, J. High-solution frequency-wavenumber spectrum analysis. Proceedings of the IEEE, 1969, vol. 57, no. 8, p. 1408-1418. DOI: 10.1109/PROC.1969.7278

[3] SCHMIDT, R. O. Multiple emitter location and signal parameter estimation. IEEE Transactions on Antennas and Propagation, 1986, vol. 34, no. 3, p. 276-280. DOI: 10.1109/TAP.1986.1143830

[4] ROY, R., PAULRAJ A., KAILATH, T. ESPRIT-A subspace rotation approach to estimation of parameters of cisoids in noise. IEEE Transactions on Acoustics, Speech, and Signal Processing, 1986, vol. 34, no. 5 , p. 1340-1342. DOI: 10.1109/TASSP.1986.1164935

[5] DONOHO, D. L. Compressive sensing. IEEE Transactions on Information Theory, 2006, vol. 52, no. 4, p. 1289-1306. DOI: $10.1109 /$ TIT.2006.871582

[6] ZHOU, C., GU, Y., ZHANG, D., et al. Compressive sensing-based coprime array direction-of-arrival estimation. IET Communications, 2017, vol. 11 , no. 11 , p. 1719-1724. DOI: $10.1049 /$ iet-com.2016.1048

[7] CARLIN, M., ROCCA, P., OLIVERI, G., et al. Directions-of-arrival estimation through Bayesian compressive sensing strategies. IEEE Transactions on Antennas and Propagation, 2013, vol. 61, no. 7, p. 3828-3838. DOI: 10.1109/TAP.2013.2256093

[8] WANG, B., ZHANG, Y. D., WANG, W. Robust DOA estimation in the presence of miscalibrated sensors. IEEE Signal Processing Letters, 2017, vol. 24, no. 7, p. 1073-1077. DOI: 10.1109/LSP.2017.2708659

[9] HUANG, Z., WANG, W. Robust measurement matrix design based on compressed sensing for DOA estimation. Radioengineering, 2019, vol. 28 , no. 1 , p. 276-282. DOI: 10.13164/re.2019.0276

[10] QI, B., WANG, W., WANG, B. Off grid compressive channel estimation for mm-wave massive MIMO with hybrid precoding. IEEE Communications Letters, 2019, vol. 23, no. 1, p. 108-111. DOI: 10.1109/LCOMM.2018.2878557

[11] SHI, Z., ZHOU, C., GU, Y., GOODMAN, N. A., QU, F. Source estimation using coprime array: A sparse reconstruction perspective. IEEE Sensors Journal, 2017, vol. 17, no. 3, p. 755-765. DOI: 10.1109/JSEN.2016.2637059

[12] LIU, Q., SO, H. C., GU, Y. Off-grid estimation with nonconvex regularization via joint sparse representation. Signal Processing, 2017, vol. 140, p. 171-176. DOI: 10.1016/j.sigpro.2017.05.020

[13] MALiOUTOV, D., CETIN, M., WHILlSKY, A. S. A sparse signal reconstruction perspective for source localization with sensor arrays. IEEE Transactions on Signal Processing, 2005, vol. 53, no. 8, p. 3010-3022. DOI: 10.1109/TSP.2005.850882

[14] CHI, Y., SCHARF, L. L., PEZESHKI, A., et al. Sensitivity to basis mismatch in compressed sensing. IEEE Transactions on Signal Processing, 2011, vol. 59, no. 5, p. 2182-2195. DOI: $10.1109 /$ TSP.2011.2112650

[15] CANDES, E., ROMBERG, J., TAO, T. Stable signal recovery from incomplete and inaccurate measurements. Communications on Pure and Applied Mathematics, 2006, vol. 59, no. 8, p. 1207-1223. DOI: $10.1002 /$ cpa.20124

[16] ZHU, H., LEUS, G., GIANNAKIS, G. Sparsity-cognizant total least-squares for perturbed compressive sampling. IEEE Transactions on Signal Processing, 2011, vol. 59, no. 5, p. 2002-2016. DOI: 10.1109/TSP.2011.2109956
[17] YANG, Z., XIE, L., ZHANG, C. Off-grid direction of arrival estimation using sparse Bayesian inference. IEEE Transactions on Signal Processing, 2013, vol. 61, no. 1, p. 38-43. DOI: 10.1109/TSP.2012.2222378

[18] WU, X., ZHU, W.P., YAN, J., ZHANG, Z. Two sparse-based methods for off-grid direction-of-arrival estimation. Signal Processing, 2018, vol. 142, p. 87-95. DOI: 10.1016/j.sigpro.2017.07.004

[19] DAI, J., BAO, X., XU, W., CHANG, C. Root sparse Bayesian learning for off-grid DOA estimation. IEEE Signal Processing Letters, 2017, vol. 24, no. 1, p. 46-50. DOI: 10.1109/LSP.2016.2636319

[20] FANG, J., WANG, F., SHEN, Y., et al. Super-resolution compressed sensing for line spectral estimation: An iterative reweighted approach IEEE Transactions on Signal Processing, 2016, vol. 64, no. 18, p. 4649-4662. DOI: 10.1109/TSP.2016.2572041

[21] TANG, G., BHASKAR, B. N., SHAH, P., RECHT, B. Compressed sensing off the grid. IEEE Transactions on Information Theory, 2013, vol. 59, no. 11, p. 7465-7490. DOI: 10.1109/TIT.2013.2277451

[22] WU, X., ZHU, W., YAN, J. A fast gridless covariance matrix reconstruction method for one- and two-dimensional direction-ofarrival estimation. IEEE Sensors Journal, 2017, vol. 17, no. 15, p. 4916-4927. DOI: 10.1109/JSEN.2017.2709329

[23] PANAHI, A., VIBERG, M., HASSIBI, B. A numerical implementation of gridless compressed sensing. In Proceedings of the 40th IEEE International Conference on Acoustics, Speech and Signal Processing (ICASSP). Brisbane (Australia), 2015, p. 3342-3346. DOI: 10.1109/ICASSP.2015.7178590

[24] STOICA, P., NEHORAI, A. MUSIC, Maximum Likelihood, and Cramér-Rao bound. IEEE Transactions on Acoustics, Speech, and Signal Processing, 1989, vol. 37, no. 5, p. 720-741. DOI: $10.1109 / 29.17564$

[25] TROPP, J. A., GILBERT, A. C. Signal recovery from random measurements via orthogonal matching pursuit. IEEE Transactions on Information Theory, 2007, vol. 53, no. 12, p. 4655-4666. DOI: $10.1109 /$ TIT.2007.909108

[26] TIBSHIRANI, R. Regression shrinkage and selection via the Lasso. Journal of Royal Statistical Society: Series B (Methodological), 1996, vol. 58, no. 1, p. 267-288. DOI: 10.1111/j.2517-6161.1996.tb02080.x

\section{About the Authors ...}

Zhenyu WEI was born in Taizhou, Jiangsu. He received his Bachelor degree from Harbin Engineering University (HEU) in 2014, Heilongjiang, China. He is currently working on his Ph.D. degree for Navigation, Guidance and Control in the College of Automation, Harbin Engineering University (HEU). His general research interests lie in the areas of wireless navigation system and array signal processing.

Xin LI (corresponding author) received Ph.D. in Pattern Recognition and Artificial Intelligence from Harbin Engineering University, China, 2011. Postdoctoral research associate at Harbin Engineering University, China (July 2006 to April 2009). Now, she is a Lecture at Harbin Engineering University. She has published about 30 refereed journal and conference papers. Her current research interests lie in the wireless navigation system and array signal processing. 
Ben WANG was born in 1990. He received his Bachelor degree from Harbin Engineering University (HEU) in 2013, Heilongjiang, China. He is currently working on his Ph.D. degree for Technology of Wireless Guidance in the Department of Automation, Harbin Engineering University (HEU). His research interests in array signal processing and MIMO radar signal processing.

Wei WANG (corresponding author) received Ph.D. in Navigation, Guidance and Control from Harbin Engineering University (HEU), Heilongjiang, China, 2005. Postdoctoral research associate at Harbin Institute of Technology, China (July 2006 to April 2009). Associate Professor at Harbin Engineering University (August 2008 to August 2010) and Academic Visitor at Loughborough University, UK (January 2010 to December 2010). Professor at Harbin Engineering University (September 2011 to now). He has published about more than 80 referred journal and conference papers.
He is a Senior Member of the Institution of Electrical and Electronic Engineers (IEEE). His current research interests include Signal Processing for Wireless Navigation Systems and MIMO Radar.

Qi LIU received his B.E. degree in Measuring \& Control Technology and Instrumentations and M.Sc. Degree in Control Science and Engineering from College of Automation, Harbin Engineering University (HEU), Harbin, China, in 2013 and 2016, respectively. He is working towards the Ph.D. degree from Department of Electronic Engineering, City University of Hong Kong (CityU), Hong Kong. From Oct. 2018 to Apr. 2019, he was a visiting scholar at the Department of Electrical and Computer Engineering, University of California, Davis (UCD). His research interests broadly lie in robust sparse approximation, array signal processing, machine learning and their applications to DOA estimation, image processing/inpainting and MIMO radar localization. 